\title{
Efficacy and Cost Effectiveness of Universal Preoperative Iron Studies in Total Hip and Knee Arthroplasty
}

Viju Daniel Varghese ( $\square$ viju.daniel@gmail.com )

Christian Medical College Vellore https://orcid.org/0000-0001-9601-6637

David Liu

Gold Coast Centre for Bone and Joint Surgery

Donald Ngo

Gold Coast University Hospital

Suzanne Edwards

University of Adelaide School of Medical Sciences: The University of Adelaide Adelaide Medical School

\section{Research Article}

Keywords: Iron deficiency anaemia, Iron studies, Blood transfusion, Hip and knee replacement

Posted Date: April 22nd, 2021

DOI: https://doi.org/10.21203/rs.3.rs-441489/v1

License: (c) This work is licensed under a Creative Commons Attribution 4.0 International License.

Read Full License 


\section{Abstract \\ Background}

Prevalence of anaemia in patients planned for total hip and knee arthroplasty is about 20\%. Optimising preoperative haemoglobin levels by iron supplementation has been shown to decrease transfusion rates, complications and associated morbidity. The need for universal screening with iron studies of all elective arthroplasty patients is not clearly defined at present.

\section{Methods}

Retrospective review of 2 sequential cohorts of patients undergoing primary hip or knee arthroplasty by a single surgeon at a single centre between January 2013 and December 2017. The first group of patients underwent pre-operative iron studies only if found to be anaemic, with a haemoglobin below $12 \mathrm{~g} / \mathrm{dL}$. From January 2015 all patients irrespective of the presence of anaemia were screened with a complete iron profile before surgery. Patients with a confirmed iron deficiency were administered with intravenous iron prior to surgery. The 2 cohorts were compared with regards to blood transfusion rate post-operatively and cost efficiency for universal screening with iron studies.

\section{Results}

There was a net decrease in allogenic blood transfusion rate from 4.76-2.92\% when universal iron studies were introduced but the difference was not statistically significant. Obtaining universal preoperative iron studies is cost neutral with the price of allogenic blood transfusion in a similar cohort. We also diagnosed 5 patients with occult malignancies.

\section{Conclusions}

Universal screening with pre-operative iron studies and iron infusion in elective arthroplasty patients may reduce allogenic blood requirements and is cost neutral. An additional benefit is the potential to diagnose asymptomatic malignancies. Further studies are required to show the true benefit of universal preoperative iron screening.

\section{Introduction}

The prevalence of anaemia among patients undergoing elective total knee (TKA) and hip arthroplasty (THA) has been demonstrated to be significant, ranging between 19 to $44 \%$, and increases with age (1$3)$. One of the major risk factors for requiring allogenic blood post total joint replacement is pre-operative anaemia. Allogenic blood transfusion has been associated with increased post-operative infection rates, length of hospital stay and mortality and morbidity (3). Pre-operative detection, evaluation and correction 
of anaemia reduces the need for allogenic blood and the incidence of complications (4).The fundamental aim of blood management in THA and TKA is to avoid allogenic blood.

Iron deficiency is one of the leading causes of anaemia, accounting for one third of anaemia cases(5). However symptomatic iron deficiency can occur independent of anaemia. Iron deficiency on its own can cause lethargy, may impair preoperative haemoglobin $(\mathrm{Hb})$ optimisation and delay post op $\mathrm{Hb}$ recovery and is associated with increased post-operative fatigue and hospital stay $(6,7)$. In a study of 715 elective orthopaedic patients, $18 \%$ of patients were found to be iron deficient in the absence of anaemia(8). Over half of the body's iron is stored in $\mathrm{Hb}$, while approximately another twenty five percent is stored intracellularly as ferrous bound to ferritin protein. These two biochemical markers are important indicators for patient recovery in post-operative period as they reflect the iron levels in the body. The function of ferritin is to replenish iron stores for heme synthesis (9)

Contemporary practice in THA and TKA is to optimise red cell count in the peri-operative period using a variety of methods. These include iron supplementation; erythropoietin; and cell salvage reinfusion(10). Parenteral iron has been shown to be effective by avoiding oral compliance issues and increases $\mathrm{Hb}$ levels within 3 weeks. There is however a paucity of evidence available regarding the use of screening iron studies and intravenous iron infusion pre-operatively with its impact on rates of allogenic blood transfusion in elective orthopaedic surgery(11).

We have been utilising a blood conservation strategy algorithm at our institution, which includes preoperative iron studies in all patients planned for elective THA and TKA surgery from January 2015 (10). Patients with iron deficiency (Serum Iron $<5$ umol/I or Serum Ferritin $<100$, TSAT $<20 \%$ ) were treated with an intravenous iron infusion pre-operatively, irrespective of pre-operative $\mathrm{Hb}$ level. The primary aim of this study is to assess whether our protocol of performing universal iron studies on all patients planned for elective joint replacement surgery helped in decreasing transfusion rates. The secondary aim is to assess the cost effectiveness of preoperative iron studies in elective THA and TKA.

The study hypothesis is that routine pre-operative iron studies in patients undergoing elective THA and TKA is effective in reducing allogenic transfusion rate and is cost effective.

\section{Methods}

\section{Study design}

A retrospective review of 2 sequential cohorts of patients who underwent primary THA or TKA under a single surgeon between January 2013 and December 2017 was performed. Universal screening for iron deficiency of all patients irrespective of $\mathrm{Hb}$ level was introduced as part of the pre-operative protocol from January 2015. Prior to this only patients found to be anaemic, defined as $\mathrm{Hb}$ level less than $12 \mathrm{~g} / \mathrm{dL}$, were investigated with iron studies and this formed the comparator control group for the study. Between the 2 sequential groups of patients, no other change in patient management or surgical technique was introduced and the surgical time was comparable. Data was collected by review of the hospital records 
and surgeon's electronic patient files. Ethics approval for the study was obtained by hospital Research and Ethics Committee.

Demographics of the two study populations are shown in Table 1. The iron studies comprised serum iron, serum ferritin, serum transferrin, serum total iron binding capacity (TIBC), and serum transferrin saturation (TSAT). Patients with studies indicating iron deficiency were referred to a haematologist, who investigated the cause and administered intravenous iron infusion as appropriate pre surgery. Iron transfusions comprised a single dose of 500-1000mg intravenous Ferritin carboxy maltose, administered in a day stay unit, given a minimum 4 weeks before surgery. The primary outcome measure of the study is blood transfusion rate between the 2 cohorts. Secondary outcome measures such as pre and postoperative $\mathrm{Hb}$ levels, per operative blood loss, and rates of iron transfusion were also collected. Data in the universal iron screening cohort, regarding patients who were diagnosed to have incidental malignancies on further investigation, was also collected.

Table 1

Comparison of cohorts pre and post universal screening

\begin{tabular}{|llll|}
\hline & $\begin{array}{l}\text { 2013-2015 } \\
\text { (Pre universal screening } \\
\text { group) }\end{array}$ & $\begin{array}{l}\text { 2015-2017 } \\
\text { (Universal screening } \\
\text { group) }\end{array}$ & P-value \\
\hline Number & 420 & 514 & \\
\hline Age - mean (SD) & $71.1(9.26)$ & $71.7(9.51)$ & 0.3324 \\
\hline Gender (M:F) & $200: 220$ & $239: 275$ & 0.9805 \\
\hline Side (Right:Left) & $195: 225$ & $271: 243$ & 0.0484 \\
\hline THA:TKA & $220: 200$ & $296: 218$ & 0.9151 \\
\hline BMI - mean (SD) & $30.12(5.60)$ & $29.56(5.67)$ & 0.1317 \\
\hline Haemoglobin (pre op) - mean & $134.97 \mathrm{gm} / \mathrm{dl}(12.33)$ & $138.33 \mathrm{gm} / \mathrm{dl}(12.91)$ & 0.0001 \\
\hline SD) & $105(25 \%)$ & $180(35 \%)$ & 0.7829 \\
\hline Iron deficiency anaemia & $21(5 \%)$ & $71(13.8 \%)$ & 0.0001 \\
\hline Iron infusion rate & $20(4.8 \%)$ & $15(2.9 \%)$ & 0.0886 \\
\hline Blood Transfusion rate & & & \\
\hline
\end{tabular}

All THA were performed through an anterolateral approach in the lateral position. Uncemented acetabular and femoral components were used, with no drain. All TKA were performed using computer navigation without tourniquet, using cemented components and routine patella resurfacing. A subcutaneous suction drains outside the joint cavity was used in TKA, with the drain removed within 24 hours after surgery. Intra-operative cell salvage was used in all cases with autologous re-infusion if sufficient blood was 
salvaged. After implantation of components, topical tranexamic acid ( $3 \mathrm{gm}$ diluted in $20 \mathrm{ml} \mathrm{N} / \mathrm{saline}$ ) was instilled for 5 minutes to the surgical site prior to final lavage and closure.

Patients were ambulated on the day of surgery. Venous thromboembolism prophylaxis comprised of enoxaparin $40 \mathrm{mg}$ daily, commenced 4 hours post-operatively until discharge from hospital. Patients were discharged on aspirin for 6 weeks post-operatively. For patients on warfarin pre-operatively, this was continued during the peri-operative period. Warfarin dose was adjusted leading to surgery, aiming for an International Normalization Ratio (INR) of 2 on the day of surgery. The warfarin was restarted the night of surgery, with bridging enoxaparin if the INR fell below 2 .

Data collected included demographics, body mass index (BMI), preoperative haemoglobin (Early $\mathrm{Hb}$ ), iron parameters (Serum iron, Total Iron binding capacity, Serum Transferrin and Serum Ferritin), Haemoglobin post iron transfusion (Late $\mathrm{Hb}$ ) and post operatively (Post-op $\mathrm{Hb}$ ), perioperative blood loss, type of anesthesia, ASA grade and type of anticoagulation, total blood loss, blood collected by cell salvage and $\mathrm{Hb}$ change between pre-operative and day 2 post-operative. Anaemia in our study is defined as $\mathrm{Hb}$ less than $13 \mathrm{~g} / \mathrm{dL}$ in males and less than $12 \mathrm{~g} / \mathrm{dL}$ in females. The transfusion trigger post-operatively was $\mathrm{Hb}$ less than $8 \mathrm{~g} / \mathrm{dL}$ or symptomatic anaemia with $\mathrm{Hb}$ less than $10 \mathrm{~g} / \mathrm{dL}$ and co-existing co-morbidities

\section{Statistical methods}

Descriptive statistics for iron variables were presented for all data and by iron transfusion (performed or not performed), depending on the distribution of the data with mean and standard deviation presented for normally distributed variables. Independent t-tests were performed to compare continuous iron variables between the two cohorts.

Bivariate binary logistic regressions were performed for the outcome: blood transfusion versus demographic and blood-related predictors. A multivariable model was performed including previous iron transfusion and variables with $\mathrm{P}$ value $<0.2$ on bivariate regression.

Bivariate linear regressions were performed for the outcomes: blood loss, RBC collected and HB drop (in separate models) versus demographic and blood-related predictors. A multivariable model was performed including previous iron transfusion and variables with $P$ value $<0.2$ on bivariate regression. Assumptions of a linear model were checked by inspection of histograms and scatter 'plots of residuals and predicted values.

A cross tabulation was performed for Iron deficiency anaemia versus general anemia, with associated Chi-Square $P$ value.

\section{Results}

Nine hundred and thirty-four patients from 2013 to 2017 were included in the study. There were 420 patients in the group prior to routine iron studies (control group) and 514 in the cohort who underwent 
routine iron deficiency screening (treatment group). The overall blood transfusion rate in the control group was $20 / 420(4.8 \%)$ and in the treatment group was 15/514 (2.9\%) with 5 following THA and 10 following TKA. Table 1 shows the demographics, iron deficiency anemia rates, iron transfusion and blood transfusion rates between the 2 cohorts. There were no major/minor side effects following the iron transfusion. There was an increase in the average $\mathrm{Hb}$ levels of $0.34 \mathrm{gm} / \mathrm{dl}$ because of the universal screening protocol which was statistically significant $(p<0.0001)$. Though there was a reduction in blood transfusion rates this was not statistically significant (Chi-Square $P$ value $=0.0980$ ).

In the 514 patients in treatment group, $9.2 \%$ were detected to have anaemia of which $30 \%$ were iron deficient. Amongst the treatment cohort with normal $\mathrm{Hb}, 34.6 \%$ were found to be iron deficient (Table 2). Iron infusions were administered to 71 of $514(13.8 \%)$ patients, with $14.4 \%$ in THA and $13.5 \%$ in TKA.

Table 2

Prevalence of anaemia and iron deficiency in joint replacement patients

\begin{tabular}{|lllll|}
\hline & Iron deficiency & Iron deficiency & Total & P value \\
\hline Anaemia & Yes & No & & 0.5990 \\
\hline Yes & 12 & 27 & $39(9.24 \%)$ & \\
\hline No & 134 & 249 & $383(90.76 \%)$ & \\
Total & $146(35 \%)$ & $276(65 \%)$ & 422 & \\
\hline
\end{tabular}

Tables $3 a$ and $4 a$ outlines the distribution of iron indices, early, late and post-operative hemoglobin rates amongst the hip and knee replacement groups respectively, and in those patients, who had iron infusion as opposed to those who did not. These results show that there was a significant improvement in the $\mathrm{Hb}$ levels of around $0.3 \mathrm{gm} / \mathrm{dl}$ following the infusion protocol. The decrease in the $\mathrm{Hb}$ levels postoperatively was similar in the iron transfusion group and non-iron transfusion groups. Table $3 \mathrm{~b}$ and $4 \mathrm{~b}$ shows comparable demographics for age, BMI and blood loss for both hip and knee cohorts. 
Table 3

a. Descriptive statistics for iron variables comparing Iron transfusion group versus Non-Iron transfusion group (Total Knee Arthroplasty-TKA)

\begin{tabular}{|c|c|c|c|c|}
\hline Iron variable & $\begin{array}{l}\text { All data } \\
(\text { mean(SD)) N } \\
=296\end{array}$ & $\begin{array}{l}\text { Iron transfusion } \\
(\text { mean }(S D)) ~ N=38\end{array}$ & $\begin{array}{l}\text { No iron infusion } \\
(\operatorname{mean}(S D)) N=258\end{array}$ & $\begin{array}{l}\text { Independent } \\
\text { T-test P value }\end{array}$ \\
\hline $\begin{array}{l}\text { S Iron (5-30 } \\
\text { umol/I) }\end{array}$ & $15.5(5.1)$ & $11.9(4.1)$ & $16.1(5.0)$ & $<0.0001$ \\
\hline $\begin{array}{l}\text { Transferrin (1.9- } \\
3.1 \mathrm{gm} / \mathrm{l})\end{array}$ & $2.5(0.4)$ & $2.8(0.4)$ & $2.5(0.3)$ & 0.0013 \\
\hline $\begin{array}{l}\text { TIBC (47-77 } \\
\text { umol/l) }\end{array}$ & $62.8(8.9)$ & $69.0(10.8)$ & $61.9(8.2)$ & 0.0015 \\
\hline $\begin{array}{l}\text { Tran satrn (20- } \\
45 \%)\end{array}$ & $25.7(10.7)$ & $18.1(6.4)$ & $26.9(10.7)$ & $<0.0001$ \\
\hline $\begin{array}{l}\text { Ferritin (30-300 } \\
\text { ug/l) }\end{array}$ & $176.1(170.3)$ & $51.0(30.4)$ & 196.0 (175.0) & $<0.0001$ \\
\hline Early $\mathrm{Hb}(\mathrm{gm} / \mathrm{dl})$ & $138.81(11.63)$ & $130.29(12.22)$ & $140.18(10.95)$ & $<0.0001$ \\
\hline Late $\mathrm{Hb}(\mathrm{gm} / \mathrm{dl})$ & 138.35 (12.04) & $132.25(13.01)$ & $139.22(11.67)$ & 0.0011 \\
\hline $\begin{array}{l}\text { Post op Hb } \\
(\mathrm{gm} / \mathrm{dl})\end{array}$ & 112.18 (13.38) & $105.41(14.44)$ & $113.22(12.93)$ & 0.0009 \\
\hline $\begin{array}{l}\text { Intraoperative } \\
\text { Blood salvage } \\
\text { rate }\end{array}$ & $47.6 \%$ & $45 \%$ & $52 \%$ & $0.4211^{\star}$ \\
\hline
\end{tabular}

Table 3

b. TKA data: Descriptive statistics of demographics

\begin{tabular}{|lll|}
\hline Variable & Mean & Standard deviation \\
\hline Current Age & 72.40 & 8.47 \\
BMI & 30.20 & 5.78 \\
Blood Loss & 331.51 & 144.63 \\
RBC Collected & 176.82 & 98.54 \\
Hb,Drop & 2.65 & 0.84 \\
\hline
\end{tabular}


Table 4

a. Descriptive statistics for iron variables comparing Iron transfusion group versus Non-Iron transfusion group (Total Hip Arthroplasty-THA)

\begin{tabular}{|c|c|c|c|c|}
\hline Iron variable & $\begin{array}{l}\text { All data } \\
(\text { mean(SD)) N } \\
=216\end{array}$ & $\begin{array}{l}\text { Iron transfusion } \\
(\text { mean }(S D)) ~ N=33\end{array}$ & $\begin{array}{l}\text { No iron infusion } \\
(\operatorname{mean}(S D)) N=183\end{array}$ & $\begin{array}{l}\text { Independent } \\
\text { T-test P value }\end{array}$ \\
\hline $\begin{array}{l}\text { S Iron (5-30 } \\
\text { umol/I) }\end{array}$ & $15.4(5.2)$ & $13.1(4.7)$ & $15.9(5.1)$ & 0.0067 \\
\hline $\begin{array}{l}\text { Transferrin (1.9- } \\
3.1 \mathrm{gm} / \mathrm{l})\end{array}$ & $2.5(0.4)$ & $2.7(0.3)$ & $2.4(0.30)$ & $<0.0001$ \\
\hline $\begin{array}{l}\text { TIBC (47-77 } \\
\text { umol/l) }\end{array}$ & $62.0(8.9)$ & $68.3(8.0)$ & $60.7(8.5)$ & $<0.0001$ \\
\hline $\begin{array}{l}\text { Tran satrn (20- } \\
45 \%)\end{array}$ & $25.6(9.3)$ & $19.8(8.6)$ & $26.8(9.0)$ & 0.0001 \\
\hline $\begin{array}{l}\text { Ferritin (30-300 } \\
\text { ug/l) }\end{array}$ & 181.5 (149.2) & $59.8(54.4)$ & $208.5(150.2)$ & $<0.0001$ \\
\hline Early $\mathrm{Hb}(\mathrm{gm} / \mathrm{dl})$ & $138.95(13.64)$ & $130.39(14.05)$ & $140.64(12.95)$ & 0.0002 \\
\hline Late $\mathrm{Hb}(\mathrm{gm} / \mathrm{dl})$ & $138.30(14.05)$ & $134.12(13.23)$ & $139.08(14.10)$ & 0.0626 \\
\hline $\begin{array}{l}\text { Post op Hb } \\
(\mathrm{gm} / \mathrm{dl})\end{array}$ & $117.26(13.44)$ & 111.55 (11.33) & $118.26(13.56)$ & 0.0100 \\
\hline $\begin{array}{l}\text { Intraoperative } \\
\text { Blood salvage } \\
\text { rate }\end{array}$ & $53.2 \%$ & $60 \%$ & $50 \%$ & $0.2912^{\star}$ \\
\hline
\end{tabular}

Table 4

b. THA data: Descriptive statistics of demographics

\begin{tabular}{|lll|}
\hline Variable & Mean & Standard deviation \\
\hline Current_Age & 68.48 & 9.91 \\
BMI & 28.67 & 5.40 \\
Blood Loss & 324.78 & 133.32 \\
RBC Collected & 168.72 & 81.91 \\
Hb Drop & 2.07 & 0.85 \\
\hline
\end{tabular}

Iron infusions were found to be predictive for blood transfusions among TKR patients but not among THR patients (Tables 5, and 6). Low pre-operative $\mathrm{Hb}$ was also strongly predictive of blood transfusion rates. Other factors that were positively associated with increased perioperative blood loss and lower post op $\mathrm{Hb}$ included general anaesthesia, use of warfarin and rivaroxaban as anticoagulants, and female gender. (Table 5,6) 
Table 5

Factors affecting Blood transfusion/Blood loss in TKA

\section{Total Knee Arthroplasty data OR/Estimate $(95 \% \mathrm{Cl}) \mathrm{p}$ value Global $\mathrm{p}$ value}

Bivariate analysis*

Previous Iron transfusion 13(3, 56) 0.0007

Multivariable analysis for outcome: Blood Transfusion*

Late pre-operative Haemoglobin $0.80(0.70,0.91) * 0.0010$

Multivariable analysis for outcome: Blood Loss

Type of Anaesthesia 0.0111

Spinal/GA vs GA -111.4 (-185.6,-37.4) 0.0032

Spinal vs GA $-71.3(-135.2,-7.5) 0.0286$

Anticoagulants $<.0001$

0 vs $2 * \star-274(-417,-130) 0.0002$

0 vs $3 *$ * $-232.7(-403.0,-62.5) 0.0074$

Multivariable analysis for outcome: Haemoglobin drop

BMI -0.02(-0.04,0.0) 0.0120

Gender (Male vs Female) $-0.35(-0.56,-0.15) 0.0007$

Anticoagulants 0.0288

0 vs 2 ** $-0.67(-1.26,-0.08) 0.0249$

1 vs $2^{\star \star}-1.44(-2.41,-0.48) 0.0034$

Late pre-operative Haemoglobin $0.02(0.01,0.03)<.0001$

*Binary logistic regression - Odds Ratio (95\% confidence interval); modelling the probability that Blood Transfusion='Yes'

${ }^{*}$ Anti coag $(0=$ Clexane, $1=$ Aspirin, $2=$ Warfarin, $3=$ All Others [Rivaroxaban, Apixaban] 
Table 6

Factors affecting Blood transfusion/Blood loss in THA

\section{Total Hip Arthroplasty data OR/Estimate $(95 \% \mathrm{Cl}) \mathrm{p}$ value Global p value}

Multi variable analysis for outcome: Blood Transfusion*

Late pre-operative Haemoglobin $0.89(0.83,0.95) 0.0006$

Multivariable analysis for outcome: RBC collected

Iron transfusion Yes vs No $60.2(22.3,98.1) 0.0019$

Type of Anaesthesia

Spinal vs GA $-38.7(-68.9,-8.4) 0.0375$

Late pre-operative Haemoglobin $1.46(0.43,2.50) 0.0056$

Multivariable analysis for outcome: Blood Loss

Iron transfusion Yes vs No $85.3(11.7,158.8) 0.0231$

Anticoagulants 0.0496

0 vs 3 ** $162.6(-294.1,-31.1) 0.0153$

2 vs $3 * *-182.4(-363.1,-1.7) 0.0478$

Multivariable analysis for outcome: Hb Drop

Late pre-operative Haemoglobin $0.02(0.01,0.03)<0.0001$

*Binary logistic model - Odds ratio $(95 \% \mathrm{Cl})$ - modelling the probability that blood transfusion $=$ Yes

${ }^{* *}$ Anti coag ( 0 = Clexane, 1 = Aspirin, 2 = Warfarin, $3=$ All Others [Rivaroxaban, Apixaban]

Amongst our elective THA and TKA cohort, we diagnosed 10 patients, following further evaluation of their iron deficiency with underlying gastric causes or malignant disease which were subsequently treated prior to joint arthroplasty surgery. These are listed in Table 7.

Table 7 Pathology Diagnosed following evaluation of Iron deficiency anaemia

\begin{tabular}{|ll|}
\hline Gastric erosions/Gastric ulcer & 5 \\
\hline Bowel Cancer & 3 \\
\hline Haematological cancer & 2 (1-Myelodysplastic syndrome, \\
& 1-Chronic myeloid leukaemia) \\
\hline
\end{tabular}

For cost effectiveness of routine pre-operative iron studies, we calculated the numbers needed to treat (NNT) of our cohort as 49.06. This is derived from the formula, NNT = 1 / incidence in control groupincidence in treated group. 
For iron infusion- NNT $=71 / 514 \times 49.06=6.8$

Cost for iron infusion: 6.8x \$135 (per iron infusion) = \$918 (\$=Australian dollar/AUD)

For iron studies - NNT $=433 / 514 x 49.06=41.33$

Cost for iron studies: $41.33 \times \$ 30$ (per iron study) $=\$ 1,240$.

Total costs for iron protocol $=$ Iron infusion costs + Iron studies costs $(\$ 918+\$ 1,240)=\$ 2,158$

For Blood transfusion

$(2 \times 15 / 512) \times 49.06=2.86$ units (each patient had 2 units of blood)

Costs for Blood transfusion: $2.86 \times \$ 700($ per blood unit) $=\$ 2,004$.

Hence our universal iron screening and iron infusion protocol is cost neutral with the expense of allogenic blood transfusion $(\$ 2,158$ vs\$2,004) in this patient cohort.

\section{Discussion}

Universal screening with preoperative iron studies and iron infusion in elective THA and TKA patients in our study decreased allogenic transfusion rates post operatively but was not statistically significant. We believe part of the reason why the allogenic transfusion rate was not significant between the 2 groups is our transfusion rate was already very low in the control patients, because of our blood management protocols (12). Universal screening with iron studies was essentially equivalent cost compared to the savings from reduction in blood transfusion rates. However, we believe pre-operative iron screening is still advantageous, as our cost calculation did not factor into account possible complications and increased length of hospital stay which often result from allogenic blood transfusion.

Pre-operative anaemia occurred in 1 out of 10 patients presenting for THA and TKA, of which Iron deficiency anaemia was the cause in one third, which is similar to the results of other studies (13). Intravenous iron infusion rapidly increases the pre-operative $\mathrm{Hb}$ levels, bypasses the effects of oral compliance, and the effect of hepcidin in patients with anaemia of chronic disease. It can help to decrease allogenic transfusion rates, reduce post op $\mathrm{Hb}$ drop after surgical blood loss and hasten the post-operative recovery rate of $\mathrm{Hb}$. The average decrease in $\mathrm{Hb}$ following THA and TKA is approximately 4gm (14). In our treatment group, we were able to increase the $\mathrm{Hb}$ preoperatively by average $0.2 \mathrm{gm}$ in TKA and 0.3gm in THA cohort. Moreover, the universal screening protocol (2015-2017) significantly increased the average $\mathrm{Hb}$ by a mean of $0.3 \mathrm{gm} / \mathrm{dl}(\mathrm{p}<0.0001)$, as compared to the cohort prior to 2015.

One third of our patients who had normal $\mathrm{Hb}$ had iron deficiency, and this is similar to recent studies(15). There are 2 important ramifications of this finding. Firstly, iron deficiency on its own, even in the absence of anaemia, can cause lethargy and tiredness. This may then hinder a patient's ability to rehabilitate following surgery(16). Secondly, iron deficiency is a sign of potential malignancy, especially after the age 
of 60 . Eight $-15 \%$ of patients with iron deficiency anaemia will have a gastro intestinal (GI) malignancy (17) and colorectal carcinoma presents with iron deficiency anaemia in $50 \%$ of cases(18). More importantly patients with iron deficiency alone in the absence of anaemia have been found to have increased risk of GI malignancy after 2 years (19). Following commencement of routine iron screening in our elective THA and TKA patients, we diagnosed 5 malignancies in patients who were otherwise asymptomatic and unaware of their diagnosis. The incidental benefit of diagnosing malignancies early in these patients cannot be discounted, which would not have been possible without universal iron studies which helped us identify these patients and instigate early treatment.

As has been demonstrated in previous studies, gender(female), type of anesthesia (GA), type of surgery (THR) and low pre-operative $\mathrm{Hb}$ were associated with increased allogenic blood transfusion risk $(20,21)$. Interestingly, we were able to show that pre-operative iron deficiency correlated with blood loss and iron transfusions were a strong prognostic factor for having post op blood transfusions. This was seen only on bivariate analysis and in the TKA group. The higher blood transfusion rates among patients who underwent iron transfusion could probably be explained by the lower baseline $\mathrm{Hb}$ levels and impaired erythropoiesis. Of the 14 patients transfused, 3 had blood disorders and 4 had multiple comorbidities with anaemia of chronic disease and consequent impaired erythropoiesis, while 4 had ischemic heart disease with stents with a low transfusion trigger.

The weaknesses of this study include the retrospective design and method of data collection via medical record review. There may have been recall bias and inaccurate data entry into the medical record which could have affected the results. Our findings may not be applicable to all regions of the world or all patient ethnicities. Additionally, as the study is a single surgeon series, surgical techniques and protocols may also vary and influence the outcomes and conclusions. Finally, as our allogenic transfusion rate in the control group was already low, the study may be underpowered and hence we were unable to demonstrate statistical significance with allogenic blood transfusion rates. Nevertheless, we believe our findings still highlight several important issues and is relevant to surgeons undertaking THA and TKA, especially so at present, where the focus has shifted to short stay or day care surgery. Correcting preoperative iron deficiency anaemia, through universal iron studies may facilitate this. The study is a single surgeon series with a uniform protocol and no change in surgical pathway apart from iron studies between the 2 comparison groups.

Transfusion rates for joint replacements have been reported to be about $18 \%$ for knee and $22 \%$ for hip arthroplasties(22), but can range from $13-87 \%$, showing (23) the high variability in clinical practice. Our low allogenic transfusion rates were decreased even further by routine pre-operative iron studies and iron infusion protocol. This could also partly explain why we were not able to show a statistically significant decrease in blood transfusion rates or cost effectiveness. While Medicare cost of blood transfusion is \$700 per unit, the cost of maintaining blood bank and personnel make the actual costs of a unit of blood about 2-5 times more(24). The true cost of allogenic blood should also factor complications and increased length of hospital stay often associated. Adding hidden costs and having a larger study group might have shown cost effectiveness with our protocol. A recent systematic review investigating pre- 
operative Iron transfusions showed a definite decrease in blood transfusions, length of hospital stay and post-operative infections(25).

\section{Conclusion}

Universal screening with pre-operative iron studies and iron infusion in elective total hip and knee arthroplasty patients may reduce allogenic blood requirements and is cost neutral. Additional benefit is the potential to diagnose asymptomatic malignancies. Further prospective studies with greater power may be required to show the true benefit of universal pre-operative iron screening by factoring into account length of hospital stay, cost of complications and maintenance of the blood bank, and cost benefits of early diagnosis of occult malignancies.

\section{List Of Abbreviations}

TKA- Total knee arthroplasty, THA- Total Hip arthroplasty

Hb- Haemoglobin, TIBC- Total iron binding capacity,

TSAT- Transferrin saturation, BMI- Body mass index

ASA- American society of anesthesiologists, GA- General anesthesia

NNT- Numbers needed to treat, GI- Gastrointestinal

\section{Declarations}

\section{Ethics approval and consent to participate}

Ethics committee approval for this study was obtained from the Greenslopes Research and Ethics committee for theis study (protocol18/44) conducted at the John Flynn hospital, Queensland, Australia, on $30 / 10 / 2018$.

\section{Consent for publication}

Not applicable

\section{Availability of data and materials}

The datasets used and/or analysed during the current study are available from the corresponding author on request.

\section{Competing interests}

The authors declare they have no competing interests. 


\section{Funding}

This study did not receive any funding.

\section{Authors' contributions}

Authors VDV and DN helped in collecting, tabulating and analysing the data from the hospital records. Author DL was the primary surgeon, who conceptualised the study and developed the protocols and was a major contributor for the study. Author SE was primarily helpful in the statistical analysis. Authors VDV and DL helped in writing and reviewing the manuscript. All authors have contributed to the write up and read and approved the final manuscript.

\section{Acknowledgements}

We would like to acknowledge the medical records staff at John Flynn Hospital and the secretarial staff at the Gold Coast Bone and Joint Centre for their help in accessing the data for this study.

\section{References}

1. Muñoz M, García-Erce JA, Cuenca J, Bisbe E, Naveira E, Awge. On the role of iron therapy for reducing allogeneic blood transfusion in orthopaedic surgery. Blood transfusion = Trasfusione del sangue. 2012;10(1):8-22.

2. Beris $P$, Muñoz M, García-Erce JA, Thomas D, Maniatis A, Van der Linden P. Perioperative anaemia management: consensus statement on the role of intravenous iron. British Journal of Anaesthesia. 2008;100(5):599-604.

3. Spahn DR, M.D., F.R.C.A. Anemia and Patient Blood Management in Hip and Knee Surgery: A Systematic Review of the Literature. Anesthesiology: The Journal of the American Society of Anesthesiologists. 2010;113(2):482-95.

4. Abdullah HR, Sim YE, Hao Y, Lin GY, Liew GHC, Lamoureux EL, et al. Association between preoperative anaemia with length of hospital stay among patients undergoing primary total knee arthroplasty in Singapore: a single-centre retrospective study. 2017;7(6):e016403.

5. Guralnik JM, Eisenstaedt RS, Ferrucci L, Klein HG, Woodman RC. Prevalence of anemia in persons 65 years and older in the United States: evidence for a high rate of unexplained anemia. Blood. 2004;104(8):2263.

6. Gomez-Ramirez S, Bisbe E, Shander A, Spahn DR, Munoz M. Management of Perioperative Iron Deficiency Anemia. Acta Haematol. 2019;142(1):21-9.

7. Arcani R, Suchon P, Venton G, Soubrier C, Gaigne L, Doddoli S, et al. Efficacy of intravenous iron therapy in non-anaemic iron-deficient patients with fatigue. Neth J Med. 2020;78(1):34-6.

8. Bisbe E, Castillo J, Sáez M, Santiveri X, Ruíz A, Muñoz M. Prevalence of preoperative anemia and hematinic deficiencies in patients scheduled for elective major orthopedic surgery. Transfusion Alternatives in Transfusion Medicine. 2008;10(4):166-73. 
9. Worwood M. Ferritin in human tissues and serum. Clin Haematol. 1982;11(2):275-307.

10. David L. Blood Conservation Strategies in Total Hip and Knee Arthroplasty. Reconstructive Review. 2015;4(4).

11. Muñoz M, Gómez-Ramírez S, Kozek-Langeneker S, Shander A, Richards T, Pavía J, et al. 'Fit to fly': overcoming barriers to preoperative haemoglobin optimization in surgical patients. Br $\mathrm{J}$ Anaesth. 2015;115(1):15-24.

12. Liu D, Dan M, Martinez Martos S, Beller E. Blood Management Strategies in Total Knee Arthroplasty. Knee Surg Relat Res. 2016;28(3):179-87.

13. Jans O, Nielsen CS, Khan N, Gromov K, Troelsen A, Husted H. Iron deficiency and preoperative anaemia in patients scheduled for elective hip- and knee arthroplasty - an observational study. Vox sanguinis. 2018;113(3):260-7.

14. Pierson JL, Hannon TJ, Earles DR. A blood-conservation algorithm to reduce blood transfusions after total hip and knee arthroplasty. Journal of Bone and Joint Surgery - Series A. 2004;86(7):1512-8.

15. Munoz M, Laso-Morales MJ, Gomez-Ramirez S, Cadellas M, Nunez-Matas MJ, Garcia-Erce JA. Preoperative haemoglobin levels and iron status in a large multicentre cohort of patients undergoing major elective surgery. Anaesthesia. 2017;72(7):826-34.

16. Gómez-Ramírez S, Bisbe E, Shander A, Spahn DR, Muñoz M. Management of Perioperative Iron Deficiency Anemia. Acta Haematol. 2019;142(1):21-9.

17. Goddard AF, James MW, McIntyre AS, Scott BB. Guidelines for the management of iron deficiency anaemia. Gut. 2011;60(10):1309-16.

18. Wilson MJ, Dekker JWT, Harlaar JJ, Jeekel J, Schipperus M, Zwaginga JJ. The role of preoperative iron deficiency in colorectal cancer patients: prevalence and treatment. Int J Colorectal Dis. 2017;32(11):1617-24.

19. Ioannou GN, Rockey DC, Bryson CL, Weiss NS. Iron deficiency and gastrointestinal malignancy: a population-based cohort study. Am J Med. 2002;113(4):276-80.

20. Klement M, Peres-Da-Silva A, Nickel B, Green C, Wellman S, Attarian D, et al. What Should Define Preoperative Anemia in Primary THA? Clinical Orthopaedics and Related Research®. 2017;475(11):2683-91.

21. Yoshihara H, Yoneoka D. Predictors of Allogeneic Blood Transfusion in Total Hip and Knee Arthroplasty in the United States, 2000-2009. The Journal of Arthroplasty. 2014;29(9):1736-40.

22. Hart A, Abou Khalil J, Carli A, Huk O, Zukor D, Antoniou J. Blood Transfusion in Primary Total Hip and Knee Arthroplasty. Incidence, Risk Factors, and Thirty-Day Complication Rates. J Bone Joint Surg-Am Vol. 2014;96(23):1945-51.

23. Shander A, Javidroozi M, Ozawa S, Hare GM. What is really dangerous: anaemia or transfusion? British journal of anaesthesia. 2011;107 Suppl 1:i41-59.

24. Farmer SL, Towler SC, Leahy MF, Hofmann A. Drivers for change: Western Australia Patient Blood Management Program (WA PBMP), World Health Assembly (WHA) and Advisory Committee on 
Blood Safety and Availability (ACBSA). Best practice \& research Clinical anaesthesiology. 2013;27(1):43-58.

25. Shin HW, Park JJ, Kim HJ, You HS, Choi SU, Lee MJ. Efficacy of perioperative intravenous iron therapy for transfusion in orthopedic surgery: A systematic review and meta-analysis. PloS one. 2019;14(5):e0215427. 